\title{
UMP Kinase Regulates Chloroplast Development and Cold Response in Rice
}

\author{
Qing Dong ${ }^{\dagger}$, Ying-Xin Zhang ${ }^{\dagger}$, Quan Zhou, Qun-En Liu, Dai-Bo Chen, Hong Wang, \\ Shi-Hua Cheng, Li-Yong Cao * and Xi-Hong Shen *
}

State Key Laboratory of Rice Biology and Key Laboratory for Zhejiang Super Rice Research, China National Rice Research Institute, Hangzhou 310006, China; dongqing66job@sina.com (Q.D.); zhangyingxin@caas.cn (Y.-X.Z.); stresszhou@163.com (Q.Z.); liuqunen202@163.com (Q.-E.L.); cdb840925@163.com (D.-B.C.); wjiyinh@126.com (H.W.); chengshihua@caas.cn (S.-H.C.)

* Correspondence: shenxihong@caas.cn (X.-H.S.); caoliyong@caas.cn (L.-Y.C.);

Tel.: +86-571-6337-0233 (X.-H.S.); +86-571-6337-0329 (L.-Y.C.)

+ These authors contributed equally to this work.

Received: 30 January 2019; Accepted: 15 April 2019; Published: 29 April 2019

check for updates

\begin{abstract}
Pyrimidine nucleotides are important metabolites that are building blocks of nucleic acids, which participate in various aspects of plant development. Only a few genes involved in pyrimidine metabolism have been identified in rice and the majority of their functions remain unclear. In this study, we used a map-based cloning strategy to isolate a UMPK gene in rice, encoding the UMP kinase that phosphorylates UMP to form UDP, from a recessive mutant with pale-green leaves. In the mutant, UDP content always decreased, while UTP content fluctuated with the development of leaves. Mutation of $U M P K$ reduced chlorophyll contents and decreased photosynthetic capacity. In the mutant, transcription of plastid-encoded RNA polymerase-dependent genes, including $p s a A$, $p s b B, p s b C$ and $p e t B$, was significantly reduced, whereas transcription of nuclear-encoded RNA polymerase-dependent genes, including $r p o A, r p o B, r p o C 1$, and $r p l 23$, was elevated. The expression of $U M P K$ was significantly induced by various stresses, including cold, heat, and drought. Increased sensitivity to cold stress was observed in the mutant, based on the survival rate and malondialdehyde content. High accumulation of hydrogen peroxide was found in the mutant, which was enhanced by cold treatment. Our results indicate that the UMP kinase gene plays important roles in regulating chloroplast development and stress response in rice.
\end{abstract}

Keywords: UMP kinase; chloroplast development; abiotic stress response; rice

\section{Introduction}

Pyrimidine nucleotides are essential for plants. They are not only the building blocks for nucleic acid synthesis, but also provide precursors for a wide range of cellular components, such as sugar, polysaccharides, glycoproteins, and phospholipids [1,2]. Pyrimidine metabolism in plants can be broadly divided into four pathways: de novo synthesis that produces UMP; nucleotide inter-conversions that modify UMP to form other pyrimidines; salvaging reactions that recycle nucleosides and free bases; and catabolism that degrades pyrimidines into simple cellular metabolites [2]. Levels of pyrimidine nucleotides depend on the interaction and coordination of enzymes involved in these pathways.

More than 20 enzymes are involved in pyrimidine metabolism in plants [1,2], whereas only a few genes encoding these enzymes have been identified by molecular genetic analysis in rice. OsDHODH1 encodes a cytosolic dihydroorotate dehydrogenase in the de novo pathway, which catalyzes the conversion of dihydroorotate to form orotate. Overexpression of OsDHODH1 enhanced plant tolerance to salt and drought stresses [3]. OsNDPK2 encodes a nucleoside diphosphate kinase in the nucleotide 
inter-conversion pathway, which catalyzes the phosphorylation of NDP to form NTP. Mutation of OsNDPK2 impaired chloroplast development and increased sensitivity to salinity stress [4,5]. ST2/ALR encodes a dCMP deaminase in the nucleotide inter-conversion pathway, which catalyzes dCMP to dUMP. Mutation of ST2/ALR impaired chloroplast development and retarded growth [6,7].

Genes encoding other enzymes involved in pyrimidine metabolism have been isolated in other plant species, including carbamoylphosphate synthase, aspartate transcarbamoylase, UMP synthase, adenylate kinase, CTP synthase, nucleoside triphosphate phosphatase, apyrase, uridine kinase, uridine ribohydrolase, cytidine deaminase, and dihydropyrimidine dehydrogenase [8-19]. These genes were found to regulate seed germination, chloroplast biogenesis, development of root and seed, response to biotic and abiotic stresses, and so on. These studies illustrated that the genes involved in pyrimidine metabolism play important roles in various aspects of plant development.

The UMP kinase is a key enzyme in the nucleotide inter-conversion pathway that phosphorylates UMP to form UDP [1,2]. Recent studies revealed that UMP kinase is involved in chloroplast development in plants. Mutation of the UMP kinase gene impaired development of photosystem I and affected the accumulation of $p s a A / B$ transcripts in Arabidopsis [20]. Two mutants of the UMP kinase gene were isolated in rice, $y g l 8$ and $y l 2$, in which reduced chlorophyll contents, decreased photochemical efficiency, and abnormal chloroplast ultrastructure were observed [21,22]. However, transcription of the genes needed for chloroplast development varied between the two mutants. In the ygls mutant, the expression of most of chlorophyll biosynthesis genes and plastid genes was significantly reduced, compared with wild-type. In the yl2 mutant, the transcription levels of genes required for chloroplast development were significantly elevated or unchanged. Therefore, the exact mechanism associated with the effects of mutation of the UMP kinase gene on chloroplast development remains to be explored. In addition, several studies have implicated some of the genes involved in the pyrimidine metabolism pathway with the response of plants to abiotic stresses [3,4,12-14]. However, little is known about the role of the UMP kinase gene.

In the present study, we identified a recessive rice mutant, umpk, with pale-green leaves throughout the entire growth period. We isolated the UMPK gene with a map-based cloning strategy and demonstrated that it encodes the UMP kinase, which affected the synthesis of UDP and UTP. Mutation of UMPK disrupted chloroplast development, likely resulting from impairment of the transcription activity of the plastid-encoded RNA polymerase. In addition, mutation of UMPK increased sensitivity to cold stress, likely due to the over-accumulation of reactive oxygen species. Our results indicate that the UMP kinase gene plays important roles in chloroplast development and abiotic stress response in rice.

\section{Results}

\subsection{Phenotype Characterization of the umpk Mutant}

The umpk mutant was identified from a mutagenized population of the indicia rice variety Zhonghui 8015 (WT) treated with ethyl methanesulfonate (EMS). Leaves of umpk exhibited a pale-green phenotype throughout the entire growth period (Figure 1a). Photosynthetic pigments were measured in six-week-old WT and mutant plants. Contents of chlorophyll a and chlorophyll b clearly decreased in the mutant compared with WT, while no significant difference was detected for carotenoid between the two genotypes (Figure S1a). The light-induced P700 absorbance changes at $820 \mathrm{~nm}(\Delta \mathrm{I} / \mathrm{Io})$, the actual quantum efficiency (ФPSII), and photosynthesis rate were also measured. All of them were significantly decreased in the mutant (Figure S1b). The ultrastructure of chloroplasts was further investigated (Figure S1c). In the WT, chloroplasts showed normal morphology. However, the chloroplasts in the mutant showed an abnormal morphology, with deformed thylakoid membranes and poorly stacked grana. These findings indicated that chloroplast development was impaired in the umpk mutant. 
a
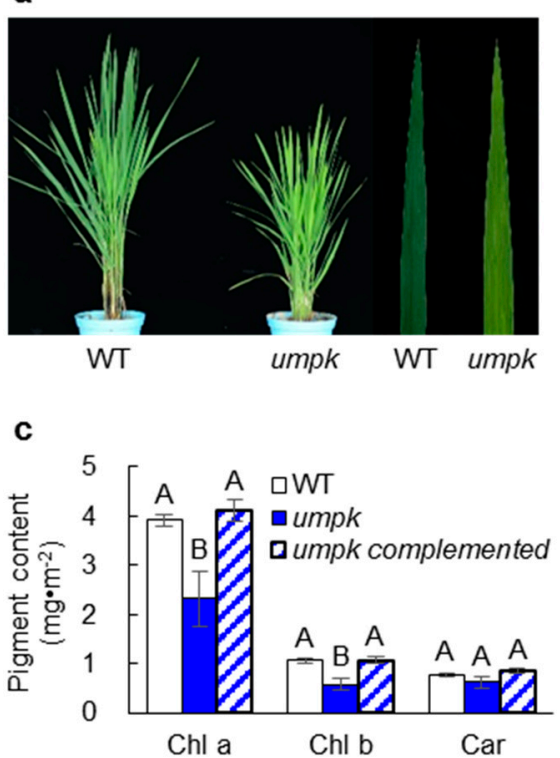

b



Figure 1. Map-based cloning of UMPK. (a) Phenotypes of the two-month-old wild type (WT) and mutant. (b) Map-based cloning of UMPK locus. (c) Chlorophyll contents of the WT, the mutant, and transgenic lines. Data represent means $\pm \mathrm{sd}(n=5)$. Bars with different letters are significantly different at $p<0.01$ based on Duncan's multiple range tests.

\subsection{Map-based Cloning of the UMPK Gene}

The umpk mutant was crossed with cultivar 02428. The resultant $\mathrm{F}_{2}$ population segregated in a 3 normal: 1 mutant ratio (green: pale green $=774: 272, \chi^{2}=0.51<\chi^{2}{ }_{0.05}=3.84, p=0.4534$ ), suggesting that the umpk mutant phenotype was due to a single recessive nuclear gene. Bulked segregant analysis revealed that four markers on the long arm of chromosome 1 co-segregated with the phenotype of umpk (Figure $1 \mathrm{~b}$ ). Plants with the recessive phenotype, including $845 \mathrm{~F}_{2}$ plants and $890 \mathrm{~F}_{3}$ plants, were further genotyped. The UMPK gene was finally narrowed down to a $121.5-\mathrm{kb}$ region flanked by markers Q1 and Q5.

This region contains 18 annotated genes (Figure 1b), according to the Rice Genome Annotation Project Database (http://rice.plantbiology.msu.edu). The coding regions of these genes were amplified from the WT and the mutant, and then sequenced. An 8-bp deletion was found in the fifth exon of LOC_Os01g73450 in the mutant, leading to a frame shift. LOC_Os01g73450, also known as $Y G L 8 / Y L 2$, has been previously reported to encode a UMP kinase and to be involved in regulating rice chloroplasts [21,22]. A genetic complementation experiment was performed by introducing a genomic fragment harboring the entire UMPK gene of the WT into the mutant. The phenotype and Chl contents of the mutant were restored to normal in the positive transgenic plants (Figure 1c, Figure S1d). Thus, UMPK was the same gene as YGL8/YL2.

\subsection{Nucleotide Synthesis Was Affected in the umpk Mutant}

The UMP kinase has been demonstrated to catalyze the phosphorylation of UMP to form UDP. UDP is converted to UTP and then to CTP [1,2]. Therefore, contents of these pyrimidine nucleotides were measured in young leaves of the six-week-old WT and mutant plants. Significant differences were detected for UDP and UTP, but not for UMP and CTP (Figure 2a). Compared with WT, UDP and UTP in the mutant decreased by $21.0 \%$ and $9.7 \%$, respectively. These decreases suggest that the mutation of $U M P K$ affected the pyrimidine synthesis and the influence was greater on the direct product UDP than on the indirect product UTP. Expression analyses were performed for UMPK and another gene, OsNDPK2, which encodes the nucleoside diphosphate kinase that phosphorylates UDP 
to form UTP $[4,5]$ (Figure 2b). The two genes exhibited higher expression levels in the mutant, more than twice those in the WT.

We further examined pyrimidine contents in older leaves. The UDP content was still lower in the mutant than in the WT, but the extent of reduction $(13.7 \%)$ was smaller than that observed in young leaves. In contrast to the results observed in the young leaves, the UTP content in the mutant increased compared with WT (14.3\%) (Figure 2c). Expression levels of the two pyrimidine synthesis genes, $U M P K$ and OsNDPK2, were also investigated. A significant difference was only detected for UMPK, which increased by $45.5 \%$ in the mutant compared with WT (Figure 2d). These results suggested that the umpk mutant might up-regulate the expression of genes involved in pyrimidine synthesis to remedy the influence caused by the mutation of UMPK.

a



C

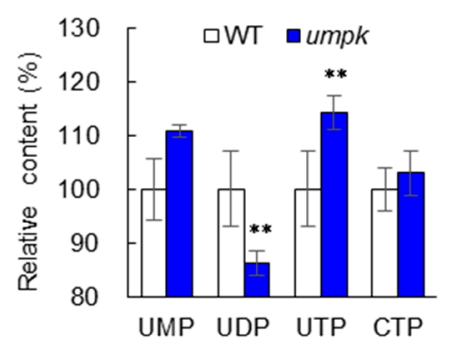

b
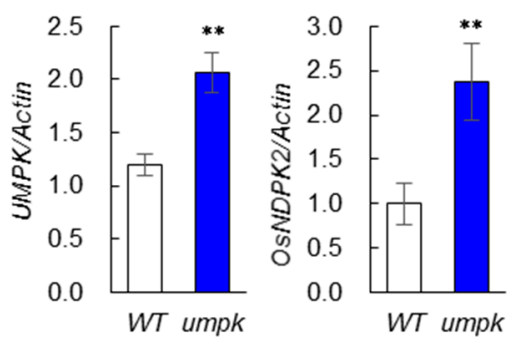

d

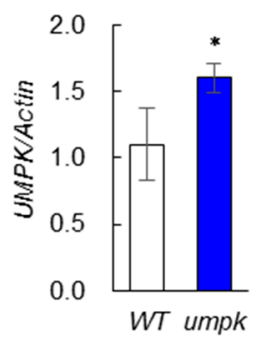

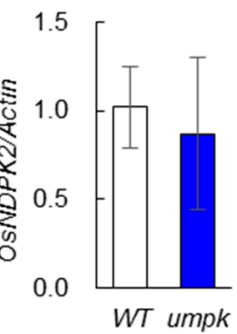

Figure 2. Pyrimidine metabolism analysis in the WT and the umpk mutant. (a) Pyrimidine nucleotides levels in the youngest fully expanded leaves. (b) Expression of two genes involved in pyrimidine metabolism in the youngest fully expanded leaves. Actin1 was used as the internal control. (c) Pyrimidine nucleotides levels in old leaves. (d) Expression of two genes involved in pyrimidine metabolism in old leaves. Actin1 was used as the internal control. Data represent means $\pm \mathrm{sd}(n=3)$. Significant differences are according to the Student's $t$-test at ${ }^{*} p<0.05$ and ${ }^{* *} p<0.01$.

\subsection{Plastid Transcription Was Altered in the umpk Mutant}

To investigate if plastid transcription was impaired by the mutation of UMPK, the expression of plastid genes was examined in young leaves of six-week-old WT and mutant plants. The genes $p s a A, p s b B, p s b C$, petB, and $r b c L$ were selected as plastid-encoded RNA polymerase (PEP)-dependent genes (Figure 3a); rpoA, rpoB, rpoC1, and $r p l 23$ were selected as nucleus-encoded RNA polymerase (NEP)-dependent genes (Figure $3 b$ ); $a t p B, n d h B$, and $p s b E$ were chosen as both PEP- and NEP-dependent genes (Figure 3c) [23-26]. The expression of all assayed PEP-dependent genes was significantly lower in the mutant, except for $r b c L$, which showed no significant difference between the WT and the mutant. Compared with WT, the expression levels of $p s a A, p s b B, p s b C$, and $p e t B$ were reduced by $54.2 \%, 34.3 \%$, $49.9 \%$, and $52.3 \%$ in the mutant, respectively. On the contrary, NEP-dependent genes were significantly up-regulated. The expression levels of $r p o A, r p o B, r p o C 1$, and $r p l 23$ in the mutant were 6.4, 5.5, 4.3, and 2.2 times greater than the WT, respectively. The genes, $\operatorname{atp} B$ and $n d h B$, which are both PEP- and NEP-dependent, were also up-regulated in the mutant. No significant difference between the WT and the mutant was observed for $p s b E$. These results demonstrate a typical plastid gene expression 
pattern caused by impaired PEP transcription, suggesting that mutation of the UMPK gene impaired transcription by the plastid-encoded RNA polymerase.

a

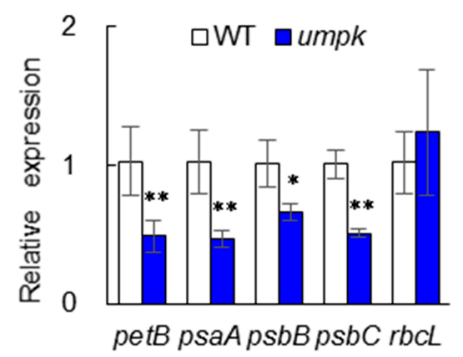

b

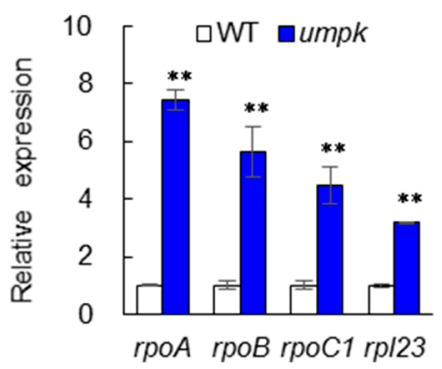

c

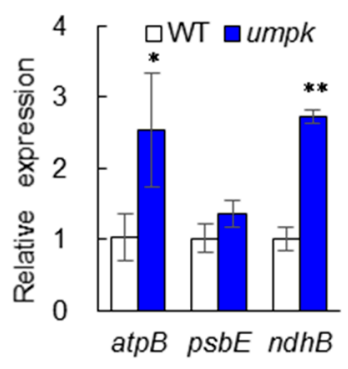

Figure 3. Expression of plastid genes in the WT and the umpk mutant. (a) Plastid-encoded RNA polymerase (PEP)-dependent genes. (b) Nucleus-encoded RNA polymerase (NEP)-dependent genes. (c) Both PEP- and NEP-dependent genes. Actin1 was used as an internal control. Data represent means $\pm \operatorname{sd}(n=3)$. Significant differences are according to the Student's $t$-test at ${ }^{*} p<0.05$ and ${ }^{* *} p<0.01$.

The expression of the plastid genes was further examined in the leaves of the one-week-old WT, the complementation plants, and the mutant plants. Compared with complementation plants, in the mutant, the four PEP-dependent genes were significantly down-regulated, while all the NEP-dependent genes were significantly up-regulated (Figure S2). These results confirmed that the transcription of plastid-encoded genes was impaired by mutation of UMPK.

\subsection{Sensitivity to Abiotic Stress Increased in the umpk Mutant}

The four-week-old WT and mutant were treated with various stresses, including cold $\left(6{ }^{\circ} \mathrm{C}\right.$, 2 days), heat $\left(45^{\circ} \mathrm{C}, 1\right.$ day), and drought (20\% PEG-6000, 4 days) conditions. The transcript level of $U M P K$ was significantly elevated by all the three stresses, and the effects were much larger in the WT than the mutant (Figure 4a). Compared with normal conditions $\left(28^{\circ} \mathrm{C}\right)$, the expression of UMPK under cold, heat, and drought conditions increased by 53.8, 49.5, and 32.0 times in the WT, respectively, while it only increased by $4.4,2.9$, and 2.1 times in the mutant, respectively. Similar results were also observed in the one-week-old WT, the complementation plants, and the mutant plants. Compared with normal conditions, the expression of UMPK under the three stresses increased by 22.0-94.2 times in the WT and the complementation plants, but it only increased by 1.3-5.8 times in the mutant (Figure S3a). These results suggest that the expression of UMPK was induced by various abiotic stresses, and this transcriptional response was impaired in the mutant.

The four-week-old WT and mutant plants subjected to cold treatment were transferred to normal conditions and survival rates were investigated after two weeks (Figure $4 \mathrm{~b}$ ). Compared with WT, survival rate decreased by $56.2 \%$ in the mutant, indicating that the mutation of UMPK increased cold sensitivity. Malondialdehyde (MDA) and hydrogen peroxide $\left(\mathrm{H}_{2} \mathrm{O}_{2}\right)$ were measured under normal and cold conditions (Figure 4c,d). Significant differences were detected between the WT and the mutant, which was larger under cold conditions than normal conditions. Compared with the WT, the level of MDA in the mutant was higher by $31.4 \%$ under control conditions, while the level was higher by $63.1 \%$ under cold conditions. The level of $\mathrm{H}_{2} \mathrm{O}_{2}$ in the mutant was higher by $110.5 \%$ compared with WT under control conditions, but it was higher by $152.1 \%$ under cold conditions.

Activities of scavengers of reactive oxygen species (ROS), including superoxide dismutase (SOD) and peroxidase (POD), were determined (Figure 4e). For POD, significant differences were detected between the WT and the mutant under the respective cold-stress and control conditions. Compared with the WT, the activities of POD in the mutant were higher by $83.4 \%$ and $74.2 \%$ under normal and cold conditions, respectively. For SOD, a significant difference between the two genotypes was only detected under cold conditions. The activity was higher by $100.1 \%$ in the mutant compared to the WT. The expression levels of the two cold-stress response genes, DREB1B and MYBS3 [27-29], 
were investigated (Figure 4f). Under control conditions, the expression levels of the two genes were significantly higher in the mutant than the WT. Conversely, they were significantly lower in the mutant than in the WT under cold conditions.

a



b

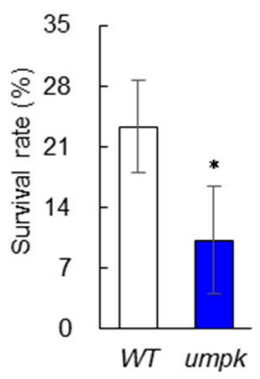

c

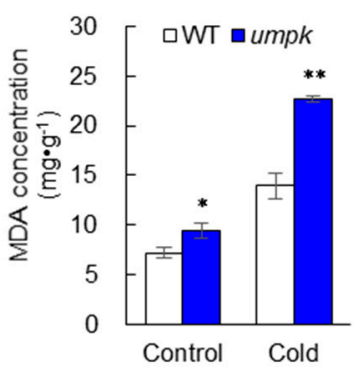

d

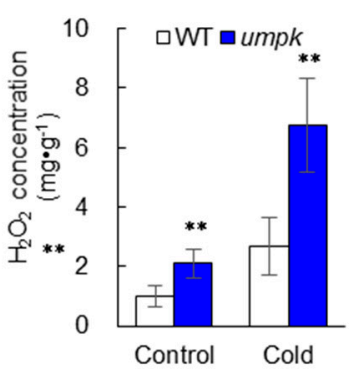

e

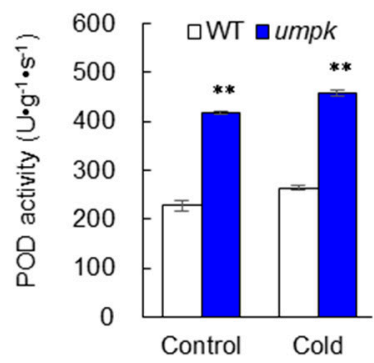



\section{f}



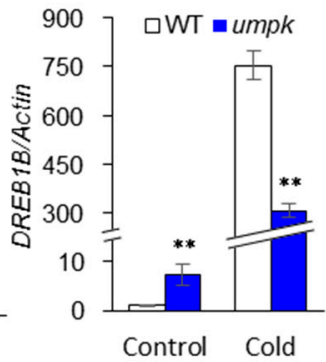

Figure 4. Response of $U M P K$ to abiotic stresses. (a) Expression levels of UMPK under various stress treatments in the WT and the mutant $(n=3)$. Actin1 was used as the internal control. (b) Survival rates of the WT and the mutant after cold treatment ( 3 replicates, 30 plants in each replicate). (c) Concentrations of malondialdehyde (MDA) in the WT and the mutant under normal and cold conditions $(n=5)$. (d) Concentrations of hydrogen peroxide $\left(\mathrm{H}_{2} \mathrm{O}_{2}\right)$ in the WT and the mutant under normal and cold conditions $(n=5)$. (e) Activities of peroxidase (POD) and superoxide dismutase (SOD) in the WT and the mutant under normal and cold conditions $(n=5)$. (f) Expression levels of two cold-inducible genes in the WT and the mutant under normal and cold conditions $(n=3)$. Actin 1 was used as the internal control. Data represent means $\pm \mathrm{sd}$. Significant differences are according to the Student's $t$-test at ${ }^{*} p<0.05$ and ${ }^{* *} p<0.01$.

\section{Discussion}

In the present study, we identified a recessive rice mutant, umpk, which displayed pale-green leaves throughout its entire growth cycle. Map-based cloning showed that UMPK encoded a UMP kinase involved in nucleotide metabolism, corresponding to $u m p k$, and was the same gene as the YGL8/YL2 genes previously reported by Zhu et al. [21] and Chen et al. [22]. These two studies revealed that mutation of the UMP kinase gene reduced Chl contents, led to abnormal ultrastructure of chloroplasts, and impaired photosynthetic capacity. These effects were also observed in our study, which demonstrates that the UMP kinase is essential for chloroplast development.

The mutations of YGL8 and YL2 exhibited some distinct molecular features. The transcription levels of most nucleus- and plastid-encoded photosynthetic genes were significantly reduced in the ygl8 mutant compared with WT; however, the expression levels of those genes in the yl2 mutant were elevated or unchanged. Expression of plastid genes depends on the activity of two types of RNA polymerases: PEP transcribes genes that are largely involved in photosynthesis, whereas NEP mainly transcribes housekeeping genes [30,31]. In the present study, the expression levels of plastid genes were investigated. The results showed that transcript levels of PEP-dependent genes and NEP-dependent genes in the umpk mutant were significantly reduced and elevated, respectively, compared with WT and the complementation lines. This is a typical plastid gene expression pattern resulting from impaired 
PEP transcription, which frequently results in abnormal chloroplast development [23,26,32-37]. The influence of impaired PEP activity on chloroplast development varied during the development of plants: it was more severe at the early stage and gradually diminished with plant development. Phenotypic changes during plant development were also observed in the yl2 mutant [22]. Therefore, the differences in molecular features between these three allelic mutations might be the result of sampling at different development stages. Our results revealed that UMPK plays an important role in maintaining PEP transcription activity and the inefficiency of chloroplast development in the umpk mutant might be associated with the impairment of PEP activity.

Three other genes involved in nucleotide metabolism were also found to regulate PEP activity. They included OsNDPK2 [5]; ATase2 encoding glutamine phosphoribosyl pyrophosphate amidotransferase that catalyzes the first step of purine biosynthesis [26]; and GARS encoding a glycinamide ribonucleotide synthetase that catalyzes the second step of purine biosynthesis [33]. In mutants of these genes, the expression of PEP-dependent genes was consistently down-regulated. Since pyrimidine and purine nucleotides are substrates for nucleic acid synthesis, it was speculated that the down-regulation of PEP-dependent genes is caused by a shortage of nucleotides in these mutants. However, up-regulation of NEP-dependent genes was also detected in the umpk mutant in the present study and the mutant of ATase2 [26]. Therefore, the relationship between the genes involved in nucleotide metabolism and plastid transcription may be more complicated.

More recently, the UMP kinase in Arabidopsis was found to be an RNA binding protein that has an impact on the accumulation of its plastid RNA targets. One of its targets, trnG-UCC, is essential for translation. Mutation of the UMP kinase gene led to a decrease of trnG-UCC, which in turn impaired the translation of chloroplast genes [38]. In the present study, the decrease of PEP activity in the umpk mutant might be caused by insufficient translation, because the core subunits of PEP are encoded by the chloroplast genes, including $r p o A, r p o B, r p o C 1$, and $r p o C 2$. For the increase of NEP activity in the umpk mutant, which is typically observed in PEP mutants $[23,26,32-37]$, there are at least two alternative explanations. First, the core subunits of NEP are encoded by nucleus genes; thus, NEP activity could not be affected by insufficient translation in chloroplasts. Conversely, the umpk mutant might up-regulate NEP activity to compensate for the decrease of PEP activity, because the core subunits of PEP are mainly transcribed by NEP. Second, RNA polymerase usage is found to switch from NEP to PEP during chloroplast development in higher plants. High activity of NEP is observed during the early stage. With the development of chloroplasts, the activity of PEP subsequently increases. One product of PEP, tRNA Glu, could repress NEP activity, leading to the switch from NEP to PEP [39]. The impairment of PEP activity in the umpk mutant might cause tRNA ${ }^{\text {Glu }}$ shortage, leading to de-repression of NEP activity.

Pyrimidine nucleotides are one of the most fundamental cellular components and have essential functions in all living organism. The UMP kinase is a crucial enzyme of pyrimidine metabolism. The kinase activity of this enzyme in rice has also been confirmed in vitro [22]. In the present study, the substrate and products of the UMP kinase were measured in the WT and the umpk mutant. The results showed that the UDP level was clearly reduced in the umpk mutant compared with WT, suggesting that UMPK encodes the enzyme that phosphorylates UMP to yield UDP. In the nucleotide inter-conversion pathway, UDP is further converted to UTP, which is catalyzed by nucleoside diphosphate kinases [1,2]. Our results showed that expression levels of UMPK and a nucleoside diphosphate kinase gene OsNDPK2 were higher in young leaves of the umpk mutant. Consistently, the magnitude of reduction of UDP was decreased, and UTP was even highly expressed in old leaves of the mutant. These findings suggest that pyrimidine metabolism is regulated by an intricate network in rice, which equilibrates different pools of nucleotides.

Several genes associated with pyrimidine metabolism are involved in the response of plants to abiotic stresses [3,4,12-14], but little is known about the role of the UMP kinase gene. In the present study, UMPK expression was induced by cold, heat and drought treatment, which was much more obvious in the WT. Furthermore, the mutation of $U M P K$ decreased the survival rate and enhanced 
MDA accumulation under cold conditions. In addition, the expression of the two positive regulators of cold tolerance, DREB1B and MYBS3, was significantly lower in the umpk mutant than the WT under cold conditions. Moreover, strong induction of $U M P K$ and the two cold-inducible genes were also observed in the complemented transgenic lines (Figure S2), though we did not investigate the survival rate and MDA accumulation in the transgenic lines. These results clearly indicate that UMPK is a positive factor in response to cold stress. DREB1B has been shown to respond quickly and transiently whereas MYBS3 responds slowly to cold stress in rice [29]. Low transcript levels were observed for both genes in the mutant under cold conditions, implying that UMPK is most likely crucial for responses to both shock and persistent cold stress.

ROS, such as singlet oxygen and $\mathrm{H}_{2} \mathrm{O}_{2}$, are by-products of various metabolic pathways and are also induced by stress conditions [40]. In general, lower doses of ROS act as molecular signals in response to abiotic stress, while higher concentrations of ROS act as toxic metabolic products in response to stresses with negative effects on plant development. Scavengers of ROS, such as SOD and $\mathrm{POD}$, play important roles in maintaining appropriate ROS levels. In our study, $\mathrm{H}_{2} \mathrm{O}_{2}$ was highly accumulated in the umpk mutant under normal conditions. This indicates that the UMP kinase is critical for maintaining appropriate ROS metabolism in rice, which is similar to four other enzymes involved in nucleotide metabolism, including nucleoside diphosphate kinase 1, nucleoside diphosphate kinase 2 , glutamine phosphoribosyl pyrophosphate amidotransferase 2, and allantoinase [4,41-43]. In addition to the high accumulation of ROS, high activities of SOD and POD were also observed in the mutant under normal conditions. The activities of SOD and POD increased but the mutant still had higher ROS accumulation, implying that the mutant cannot produce sufficient ROS scavengers to reduce the ROS even under normal conditions. When the mutant was exposed to cold stress, a further increase in ROS consequently led to increased sensitivity of the umpk mutant to cold stress. Therefore, the over-accumulation of ROS caused by low levels of UMP kinases might be a reason why the sensitivity to cold stress increased in the umpk mutant.

Chloroplasts are the main site of ROS production in plants and act as sensors of environmental stresses [40]. An increase of ROS levels has been frequently observed in mutants with impaired chloroplast functions [4,41-43]. Accordingly, the over-accumulation of ROS and increase of cold sensitivity in the umpk mutant might be an indirect effect of abnormal chloroplast ultrastructure and photosynthetic activity. However, we cannot rule out the possibility that UMPK regulates ROS production through another pathway. It should be noted that the expression of NDPK2 increased in the umpk mutant. Arabidopsis AtNDPK2 has been implicated in ROS signaling through its interaction with ROS regulators, including AtMPK3, AtMPK6, and SOS2 [44,45]. Therefore, UMPK could be involved in the regulation of ROS levels through regulation of pyrimidine metabolism. Further biochemical and molecular studies are required to elucidate the exact roles of UMPK in the regulation of ROS accumulation and cold stress response.

\section{Materials and Methods}

\subsection{Plant Material and Growth Conditions}

The umpk mutant was obtained from a mutant population of indica rice cultivar Zhonghui 8015 after EMS treatment. For the EMS treatment, seeds soaked for $12 \mathrm{~h}$ in water were transferred into a $1.0 \%$ EMS solution for $12 \mathrm{~h}$, followed by a $12 \mathrm{~h}$ wash using flowing water to accelerate germination. Rice materials were tested in the experimental stations of the China National Rice Research Institute located at Hangzhou in Zhejiang Province. For experiments (except for abiotic stress treatments), rice lines were grown in a paddy field following standard management practices. For abiotic stress treatments, rice lines were grown in hydroponic culture with a nutrient solution in a chamber with 12-h light/12-h dark cycles. The composition of the nutrient solution was the same as described in Yoshida et al. [46]. Light was provided by fluorescent lamps with $220 \mu \mathrm{mol} \mathrm{m}^{-2} \mathrm{~s}^{-1}$ of light intensity. The plants were grown at $28^{\circ} \mathrm{C}$ for about 4 weeks (WT and mutant plants) or about 5 days (WT, complementation 
plants, and mutant plants), and then treated with abiotic stresses, including cold (seedlings were transferred to $6{ }^{\circ} \mathrm{C}$ for 2 days), heat (seedlings were transferred to $45^{\circ} \mathrm{C}$ for 1 day), and drought (20\% PEG-6000 was added to the hydroponic culture medium for 4 days).

\subsection{Measurement of Pigment Content, Photochemical Efficiency of Photosystem, and Photosynthetic Rate, and Transmission Electron Microscopy Assay}

The youngest fully expanded leaves from the six-week-old WT and mutant were used. The contents of pigments, including $\mathrm{Chl}$ a, Chl b, and carotenoid, were determined using a spectrophotometer (DU800, Beckman, Brea, California, USA), according to the method of Lichtenthaler [47]. The photochemical capacity of PSI was determined as $\Delta \mathrm{I} / \mathrm{I}$, according to the method of Zhang et al. [48]. The photochemical capacity of PSII was determined as $\Phi P S I I$ using a PAM-2500 portable chlorophyll fluorometer (Heinz Walz, Forchheim, Germany), according to the method of Zhao et al. [49]. The photosynthetic rate was determined at 9:00-11:00 on a sunny day using the portable photosynthesis measurement device LI-6400 (LICOR, Lincoln, Nebraska, USA), according to the method of Zhao et al. [49]. Transmission electron microscopy was performed according to the method of Li et al. [50].

\subsection{Map-based Cloning of UMPK}

The umpk mutant was crossed with rice cultivar 02428 to construct a segregating population. For genetic tests, 1046 plants were randomly selected from the $F_{2}$ population containing more than 3000 individuals for phenotype analysis. For preliminary mapping, a bulk segregant analysis method [51] was initially used. The mutant and 02428 were assayed with 436 SSR markers and 91 Indel markers, and a total of 110 markers showing polymorphism between the two varieties were identified. Two phenotypic bulks, one consisting of 10 individuals with the WT phenotype and another consisting of 10 individuals with the pale green phenotype, were selected from the $F_{2}$ population. The two bulks were assayed with the polymorphic markers to determine if the markers co-segregated with the phenotype of umpk. To validate these co-segregating markers, $36 \mathrm{~F}_{2}$ plants with the recessive pale green phenotype were genotyped. For fine-mapping, additional polymorphic InDel markers between the mutant and 02428 were developed to assay an additional $809 \mathrm{~F}_{2}$ plants; $890 \mathrm{~F}_{3}$ plants with the recessive pale green phenotype were assayed with the markers surrounding the preliminary linkage locus.

To analyze sequences of annotated genes located in the fine-mapping region, the coding regions of these genes were amplified from the WT and the mutant, and then sequenced. For complementation, a 6916 bp genomic fragment containing an entire open reading frame, $1519 \mathrm{bp}$ upstream of the start codon, and $1616 \mathrm{bp}$ downstream of the termination codon for UMPK was amplified from the WT. The PCR product was recombined into the binary vector pCAMBIA1300 using an In-Fusion Advantage Cloning kit (Takara, Japan). The resultant expression construct was introduced into the umpk mutant by Agrobacterium-mediated transformation. SSR markers were selected from the Gramene database (http://www.gramene.org). The InDel markers used for bulk segregant analysis were selected from the study of $\mathrm{Wu}$ et al. [52]. The InDel markers used for fine-mapping were developed according to differences between the genome sequence of japonica Nipponbare and indica 9311 (http://www.gramene.org). The primers used for sequence analysis and construction of complementation vector were developed according to the sequence of Nipponbare. The primers developed in this study are listed in Table S1.

\subsection{RNA Extraction and Quantitative Real-Time PCR Analysis}

To investigate the expression of pyrimidine synthesis genes, young leaves (the youngest fully expanded leaves) and old leaves (the fourth leaf from the top) were collected from the six-week-old WT and mutant. To investigate the expression levels of plastid genes, young leaves were collected from the six-week-old WT and mutant, or leaves were collected from the one-week-old WT, complementation lines, and the mutant. To examine the expression of $U M P K$ or cold-inducible genes under normal and abiotic stresses conditions, young leaves were collected from the four-week-old WT and mutant, 
or leaves were collected from about one-week-old WT plants, complementation lines, and the mutant. Total RNA was extracted from these rice tissues using an RNeasy Plus Mini Kit (QIAGEN, German). First-strand cDNA was synthesized using a ReverTra AceR kit (Toyobo, Japan). Quantitative real-time PCR was performed on an Applied Biosystems 7500 using SYBR qPCR Mix kit (Toyobo, Japan). Actin1 was used as the endogenous control. Data were analyzed according to the $2^{-\Delta \Delta C t}$ method [53]. Three independent biological replicates, each consisting of five plants for tissue samples, and three technical replicates per biological replicate were used. Primers are listed in Table S1.

\subsection{Nucleotide Measurement}

New leaves (the youngest fully expanded leaves) and old leaves (the fourth leaf from the top) were collected from the six-week-old WT and mutant. Samples were frozen immediately in liquid nitrogen and homogenized with phosphate buffered saline ( $\mathrm{pH}$ 7.4) containing $81 \mathrm{mM} \mathrm{Na}_{2} \mathrm{HPO}_{4}$ and $19 \mathrm{mM} \mathrm{NaH}_{2} \mathrm{PO}_{4}$. The contents of UMP, UDP, UTP, and CTP were examined by corresponding ELISA kits (Mlbio, Shanghai, China) using a micro-ELISA reader RT-6100 (Rayto, Shenzhen, China) equipped with a $450 \mathrm{~nm}$ filter.

\subsection{Measurement of $\mathrm{MDA}$ and $\mathrm{H}_{2} \mathrm{O}_{2}$ Contents}

The youngest fully expanded leaves were collected from four-week-old rice lines grown in chambers with normal conditions or stress treatment conditions. For measurement of MDA contents, samples were ground in liquid nitrogen using a mortar and pestle into which $5 \mathrm{~mL}$ ice-cold $10 \%$ $(w / v)$ trichloroacetic acid had been added. The concentration was measured following the method of Dionisio-Sese and Tobita [54]. For measurement of $\mathrm{H}_{2} \mathrm{O}_{2}$ contents, samples were homogenized in $4 \mathrm{~mL}$ of $10 \mathrm{mM}$ 3-amino-1, 2, 4-triazole, and the samples were centrifuged for $25 \mathrm{~min}$ at $6000 \mathrm{~g}$. The supernatant solution was used to determine the concentration following the method of Brennan and Frenkel [55].

\subsection{Determination of Antioxidant Enzyme Activities}

The youngest fully expanded leaves were collected from four-week-old rice lines grown in chambers with normal conditions or stress treatment conditions. Samples were homogenized in a $5 \mathrm{~mL}$ extraction buffer ( $100 \mathrm{mM}$ sodium phosphate buffer, $\mathrm{pH}$ 7.0). The homogenates were centrifuged for $15 \mathrm{~min}$ at $10,000 \times \mathrm{g}$ at $4{ }^{\circ} \mathrm{C}$, and then the supernatant solution was collected. The SOD activity was assayed based on inhibition of photoreduction of nitroblue tetrazolium as described by Giannopolitis and Ries [56]. The POD activity was assayed based on the conversion of guaiacol to tetraguaiacol, which was monitored at $470 \mathrm{~nm}$ as described by Maehly and Chance [57].

\section{Conclusions}

Our study revealed that $U M P K$, encoding the UMP kinase, is involved in the regulation of PEP-dependent gene transcription and is essential for chloroplast development. Moreover, mutation of $U M P K$ leads to over-accumulation of reactive oxygen species and consequently increases sensitivity to cold stress. These results enhance our understanding of the roles of the UMP kinase gene in chloroplast development and abiotic stress response in rice.

Supplementary Materials: Supplementary materials can be found at http://www.mdpi.com/1422-0067/20/9/2107/ s1.

Author Contributions: X.-H.S. and L.-Y.C. conceived and designed the experiments; Y.-X.Z. constructed the mapping population; Q.D. and Q.-E.L. performed laboratory experiments; Q.D., Q.Z., D.-B.C, and H.W. performed the field experiments; Q.D. analyzed the data and drafted the manuscript; Q.D., X.-H.S., L.-Y.C., S.-H.C., and Y.-X.Z. reviewed and edited the manuscript. All authors read and approved the final manuscript.

Funding: This work was supported by the National Key Transgenic Research Project (2016ZX08001-002), the Agricultural Science and Technology Innovation Program of the Chinese Academy of Agricultural Science (CAAS-ASTIP-2013-CNRRI), and the Natural Science Foundation of the Innovation Research Group (31521064). 
Conflicts of Interest: The authors declare no conflicts of interest.

$\begin{array}{ll}\text { Abbreviations } \\ \text { Chl } & \text { Chlorophyll } \\ \text { Car } & \text { Carotenoid } \\ \text { PSI } & \text { Photosystem I } \\ \text { PSII } & \text { Photosystem II } \\ \Delta I / I o & \text { Content of active P700 } \\ \Phi_{P S I I} & \text { Actual quantum efficiency } \\ \mathrm{CP} & \text { Chloroplast } \\ \mathrm{G} & \text { Grana } \\ \mathrm{ROS} & \text { Reactive oxygen species } \\ \mathrm{MDA} & \text { Malondialdehyde } \\ \mathrm{H}_{2} \mathrm{O}_{2} & \text { Hydrogen peroxide } \\ \mathrm{SOD} & \text { Superoxide dismutase } \\ \text { POD } & \text { Peroxidase } \\ \text { PEP } & \text { Plastid-encoded RNA polymerase } \\ \text { NEP } & \text { Nucleus-encoded RNA polymerase }\end{array}$

\section{References}

1. Zrenner, R.; Stitt, M.; Sonnewald, U.; Boldt, R. Pyrimidine and purine biosynthesis and degradation in plants. Annu. Rev. Plant Biol. 2006, 57, 805-836. [CrossRef] [PubMed]

2. Kafer, C.; Zhou, L.; Santoso, D.; Guirgis, A.; Weers, B.; Park, S.; Thornburg, R. Regulation of pyrimidine metabolism in plants. Front Biosci. 2004, 9, 1611-1625. [PubMed]

3. Liu, W.Y.; Wang, M.M.; Huang, J.; Tang, H.J.; Lan, H.X.; Zhang, H.S. The OsDHODH1 gene is involved in salt and drought tolerance in rice. J. Integr. Plant Biol. 2009, 51, 825-833. [CrossRef]

4. Ye, W.; Hu, S.; Wu, L.; Ge, C.; Cui, Y.; Chen, P.; Wang, X.; Xu, J.; Ren, D.; Dong, G.; et al. White stripe leaf 12 (WSL12), encoding a nucleoside diphosphate kinase 2 (OsNDPK2), regulates chloroplast development and abiotic stress response in rice (Oryza sativa L.). Mol. Breed. 2016, 36, 57. [CrossRef]

5. Zhou, K.N.; Xia, J.F.; Wang, Y.L.; Ma, T.C.; Li, Z.F. A Young Seedling Stripe2 phenotype in rice is caused by mutation of a chloroplast-localized nucleoside diphosphate kinase 2 required for chloroplast biogenesis. Genet. Mol. Biol. 2017, 40, 630-642. [CrossRef]

6. Niu, M.; Wang, Y.; Wang, C.; Lyu, J.; Wang, Y.; Dong, H.; Long, W.; Wang, D.; Kong, W.; Wang, L.; et al. ALR encoding dCMP deaminase is critical for DNA damage repair, cell cycle progression and plant development in rice. J. Exp. Bot. 2017, 68, 5773-5786. [CrossRef] [PubMed]

7. Xu, J.; Deng, Y.; Li, Q.; Zhu, X.; He, Z. STRIPE2 encodes a putative dCMP deaminase that plays an important role in chloroplast development in rice. J. Genet. Genom. 2014, 41, 539-548. [CrossRef] [PubMed]

8. Geigenberger, P.; Regierer, B.; Nunes-Nesi, A.; Leisse, A.; Urbanczyk-Wochniak, E.; Springer, F.; van Dongen, J.T.; Kossmann, J.; Fernie, A.R. Inhibition of de novo pyrimidine synthesis in growing potato tubers leads to a compensatory stimulation of the pyrimidine salvage pathway and a subsequent increase in biosynthetic performance. Plant Cell 2005, 17, 2077-2088. [CrossRef] [PubMed]

9. Chen, C.T.; Slocum, R.D. Expression and functional analysis of aspartate transcarbamoylase and role of de novo pyrimidine synthesis in regulation of growth and development in Arabidopsis. Plant Physiol. Biochem. 2008, 46, 150-159. [CrossRef]

10. Feng, X.; Yang, R.; Zheng, X.; Zhang, F. Identification of a novel nuclear-localized adenylate kinase 6 from Arabidopsis thaliana as an essential stem growth factor. Plant Physiol. Biochem. 2012, 61, 180-186. [CrossRef]

11. Lange, P.R.; Geserick, C.; Tischendorf, G.; Zrenner, R. Functions of chloroplastic adenylate kinases in Arabidopsis. Plant Physiol. 2008, 146, 492-504. [CrossRef] [PubMed]

12. Daumann, M.; Hickl, D.; Zimmer, D.; DeTar, R.A.; Kunz, H.H.; Mohlmann, T. Characterization of filament-forming CTP synthases from Arabidopsis thaliana. Plant J. 2018, 96, 316-328. [CrossRef] [PubMed] 
13. Cheung, M.Y.; Li, M.W.; Yung, Y.L.; Wen, C.Q.; Lam, H.M. The unconventional P-loop NTPase OsYchF1 and its regulator OsGAP1 play opposite roles in salinity stress tolerance. Plant Cell Environ. 2013, 36, $2008-2020$. [CrossRef] [PubMed]

14. Cheung, M.Y.; Li, X.; Miao, R.; Fong, Y.H.; Li, K.P.; Yung, Y.L.; Yu, M.H.; Wong, K.B.; Chen, Z.; Lam, H.M. ATP binding by the P-loop NTPase OsYchF1 (an unconventional G protein) contributes to biotic but not abiotic stress responses. Proc. Natl. Acad. Sci. USA 2016, 113, 2648-2653. [CrossRef] [PubMed]

15. Wu, J.; Steinebrunner, I.; Sun, Y.; Butterfield, T.; Torres, J.; Arnold, D.; Gonzalez, A.; Jacob, F.; Reichler, S.; Roux, S.J. Apyrases (nucleoside triphosphate-diphosphohydrolases) play a key role in growth control in Arabidopsis. Plant Physiol. 2007, 144, 961-975. [CrossRef]

16. Deng, S.; Sun, J.; Zhao, R.; Ding, M.; Zhang, Y.; Sun, Y.; Wang, W.; Tan, Y.; Liu, D.; Ma, X.; et al. Populus euphratica APYRASE2 enhances cold tolerance by modulating vesicular trafficking and extracellular ATP in Arabidopsis plants. Plant Physiol. 2015, 169, 530-548. [CrossRef] [PubMed]

17. Chen, M.; Thelen, J.J. Plastid uridine salvage activity is required for photoassimilate allocation and partitioning in Arabidopsis. Plant Cell 2011, 23, 2991-3006. [CrossRef]

18. Riegler, H.; Geserick, C.; Zrenner, R. Arabidopsis thaliana nucleosidase mutants provide new insights into nucleoside degradation. New Phytol. 2011, 191, 349-359. [CrossRef] [PubMed]

19. Cornelius, S.; Witz, S.; Rolletschek, H.; Mohlmann, T. Pyrimidine degradation influences germination seedling growth and production of Arabidopsis seeds. J. Exp. Bot. 2011, 62, 5623-5632. [CrossRef] [PubMed]

20. Hein, P.; Stockel, J.; Bennewitz, S.; Oelmuller, R. A protein related to prokaryotic UMP kinases is involved in psaA/B transcript accumulation in Arabidopsis. Plant Mol. Biol. 2009, 69, 517-528. [CrossRef] [PubMed]

21. Zhu, X.; Guo, S.; Wang, Z.; Du, Q.; Xing, Y.; Zhang, T.; Shen, W.; Sang, X.; Ling, Y.; He, G. Map-based cloning and functional analysis of $Y G L 8$, which controls leaf colour in rice (Oryza sativa). BMC Plant Biol. 2016, 16, 134. [CrossRef]

22. Chen, F.; Dong, G.; Ma, X.; Wang, F.; Zhang, Y.; Xiong, E.; Wu, J.; Wang, H.; Qian, Q.; Wu, L.; et al. UMP kinase activity is involved in proper chloroplast development in rice. Photosynth Res. 2018, 137, 53-67. [CrossRef]

23. Lv, Y.; Shao, G.; Qiu, J.; Jiao, G.; Sheng, Z.; Xie, L.; Wu, Y.; Tang, S.; Wei, X.; Hu, P. White leaf and panicle 2, encoding a PEP-associated protein, is required for chloroplast biogenesis under heat stress in rice. J. Exp. Bot. 2017, 68, 5147-5160. [CrossRef]

24. Qiu, Z.; Chen, D.; He, L.; Zhang, S.; Yang, Z.; Zhang, Y.; Wang, Z.; Ren, D.; Qian, Q.; Guo, L.; et al. The rice white green leaf 2 gene causes defects in chloroplast development and affects the plastid ribosomal protein S9. Rice 2018, 11, 39. [CrossRef]

25. Ye, L.S.; Zhang, Q.; Pan, H.; Huang, C.; Yang, Z.N.; Yu, Q.B. EMB2738, which encodes a putative plastid-targeted GTP-binding protein, is essential for embryogenesis and chloroplast development in higher plants. Physiol. Plant 2017, 161, 414-430. [CrossRef]

26. Yang, Z.; Shang, Z.; Wang, L.; Lu, Q.; Wen, X.; Chi, W.; Zhang, L.; Lu, C. Purine biosynthetic enzyme ATase2 is involved in the regulation of early chloroplast development and chloroplast gene expression in Arabidopsis. Photosynth Res. 2015, 126, 285-300. [CrossRef]

27. Dou, M.; Cheng, S.; Zhao, B.; Xuan, Y.; Shao, M. The indeterminate domain protein ROC1 regulates chilling tolerance via activation of DREB1B/CBF1 in rice. Int. J. Mol. Sci. 2016, 17, 233. [CrossRef]

28. Gutha, L.R.; Reddy, A.R. Rice DREB1B promoter shows distinct stress-specific responses, and the overexpression of cDNA in tobacco confers improved abiotic and biotic stress tolerance. Plant Mol. Biol. 2008, 68, 533-555. [CrossRef]

29. Su, C.F.; Wang, Y.C.; Hsieh, T.H.; Lu, C.A.; Tseng, T.H.; Yu, S.M. A novel MYBS3-dependent pathway confers cold tolerance in rice. Plant Physiol. 2010, 153, 145-158. [CrossRef]

30. Liebers, M.; Grubler, B.; Chevalier, F.; Lerbs-Mache, S.; Merendino, L.; Blanvillain, R.; Pfannschmidt, T. Regulatory shifts in plastid transcription play a key role in morphological conversions of plastids during plant development. Front. Plant Sci. 2017, 8, 23. [CrossRef]

31. Borner, T.; Aleynikova, A.Y.; Zubo, Y.O.; Kusnetsov, V.V. Chloroplast RNA polymerases: Role in chloroplast biogenesis. Biochim. Biophys. Acta 2015, 1847, 761-769. [CrossRef]

32. He, L.; Zhang, S.; Qiu, Z.; Zhao, J.; Nie, W.; Lin, H.; Zhu, Z.; Zeng, D.; Qian, Q.; Zhu, L. FRUCTOKINASE-LIKE PROTEIN 1 interacts with TRXz to regulate chloroplast development in rice. J. Integr. Plant Biol. 2018, 60, 94-111. [CrossRef] [PubMed] 
33. Cao, P.; Ren, Y.; Liu, X.; Zhang, T.; Zhang, P.; Xiao, L.; Zhang, F.; Liu, S.; Jiang, L.; Wan, J. Purine nucleotide biosynthetic gene GARS controls early chloroplast development in rice (Oryza sativa L.). Plant Cell Rep. 2019, 38, 183-194. [CrossRef]

34. Zhang, Z.; Cui, X.; Wang, Y.; Wu, J.; Gu, X.; Lu, T. The RNA editing factor WSP1 is essential for chloroplast development in rice. Mol. Plant 2017, 10, 86-98. [CrossRef] [PubMed]

35. Wang, Y.; Ren, Y.; Zhou, K.; Liu, L.; Wang, J.; Xu, Y.; Zhang, H.; Zhang, L.; Feng, Z.; Wang, L.; et al. WHITE STRIPE LEAF4 encodes a novel P-type PPR protein required for chloroplast biogenesis during early leaf development. Front. Plant Sci. 2017, 8, 1116. [CrossRef]

36. Lin, D.; Jiang, Q.; Ma, X.; Zheng, K.; Gong, X.; Teng, S.; Xu, J.; Dong, Y. Rice TSV3 encoding Obg-Like GTPase protein is essential for chloroplast development during the early leaf stage under cold stress. Genes Genom. Genet. 2018, 8, 253-263. [CrossRef]

37. Zhang, Y.Y.; Hao, Y.Y.; Wang, Y.H.; Wang, C.M.; Wang, Y.L.; Long, W.H.; Wang, D.; Liu, X.; Jiang, L.; Wan, J.M. Lethal albinic seedling, encoding a threonyl-tRNA synthetase, is involved in development of plastid protein synthesis system in rice. Plant Cell Rep. 2017, 36, 1053-1064. [CrossRef]

38. Schmid, L.M.; Ohler, L.; Mohlmann, T.; Brachmann, A.; Muino, J.M.; Leister, D.; Meurer, J.; Manavski, N. PUMPKIN, the Sole Plastid UMP Kinase, Associates with Group II Introns and Alters Their Metabolism. Plant Physiol. 2019, 179, 248-264. [CrossRef]

39. Hanaoka, M.; Kanamaru, K.; Fujiwara, M.; Takahashi, H.; Tanaka, K. Glutamyl-tRNA mediates a switch in RNA polymerase use during chloroplast biogenesis. EMBO Rep. 2005, 6, 545-550. [CrossRef]

40. Choudhury, F.K.; Rivero, R.M.; Blumwald, E.; Mittler, R. Reactive oxygen species, abiotic stress and stress combination. Plant J. 2017, 90, 856-867. [CrossRef]

41. Nourimand, M.; Todd, C.D. There is a direct link between allantoin concentration and cadmium tolerance in Arabidopsis. Plant Physiol. Biochem. 2019, 135, 441-449. [CrossRef]

42. Woo, N.S.; Gordon, M.J.; Graham, S.R.; Rossel, J.B.; Badger, M.R.; Pogson, B.J. A mutation in the purine biosynthetic enzyme ATASE2 impacts high light signalling and acclimation responses in green and chlorotic sectors of Arabidopsis leaves. Funct. Plant Biol. 2011, 38, 401-419. [CrossRef]

43. Dorion, S.; Clendenning, A.; Rivoal, J. Engineering the expression level of cytosolic nucleoside diphosphate kinase in transgenic Solanum tuberosum roots alters growth, respiration and carbon metabolism. Plant J. 2017, 89, 914-926. [CrossRef]

44. Moon, H.; Lee, B.; Choi, G.; Shin, D.; Prasad, D.T.; Lee, O.; Kwak, S.S.; Kim, D.H.; Nam, J.; Bahk, J.; et al. NDP kinase 2 interacts with two oxidative stress-activated MAPKs to regulate cellular redox state and enhances multiple stress tolerance in transgenic plants. Proc. Natl. Acad. Sci. USA 2003, 100, 358-363. [CrossRef]

45. Verslues, P.E.; Batelli, G.; Grillo, S.; Agius, F.; Kim, Y.S.; Zhu, J.; Agarwal, M.; Katiyar-Agarwal, S.; Zhu, J.K. Interaction of SOS2 with nucleoside diphosphate kinase 2 and catalases reveals a point of connection between salt stress and $\mathrm{H}_{2} \mathrm{O}_{2}$ signaling in Arabidopsis thaliana. Mol. Cell. Biol. 2007, 27, 7771-7780. [CrossRef]

46. Yoshida, S.; Forno, D.A.; Cock, J.H.; Gomez, K.A. Laboratory Manual for Physiological Studies of Rice, 3rd ed.; The International Rice Research Institute: Manila, Philippines, 1976.

47. Lichtenthaler, H.K. Chlorophylls and carotenoids: Pigments of photosynthetic biomembranes. Method Enzymol. 1987, 148, 350-382.

48. Zhang, Z.; Jia, Y.; Gao, H.; Zhang, L.; Li, H.; Meng, Q. Characterization of PSI recovery after chilling-induced photoinhibition in cucumber (Cucumis sativus L.) leave. Planta 2011, 234, 883-889. [CrossRef]

49. Zhao, X.; Chen, T.; Feng, B.; Zhang, C.; Peng, S.; Zhang, X.; Fu, G.; Tao, L. Non-photochemical quenching plays a key role in light acclimation of rice plants differing in leaf color. Front. Plant Sci. 2017, 7, 1968. [CrossRef] [PubMed]

50. Li, Z.; Zhang, Y.; Liu, L.; Liu, Q.; Bi, Z.; Yu, N.; Cheng, S.; Cao, L. Fine mapping of the lesion mimic and early senescence 1 (lmes1) in rice (Oryza sativa). Plant Physiol. Bioch. 2014, 80, 300-307. [CrossRef]

51. Michelmore, R.W.; Paran, I.; Kesseli, R.V. Identification of markers linked to disease-resistance genes by bulked segregant analysis: A rapid method to detect markers in specific genomic regions by using segregating populations. Proc. Natl. Acad. Sci. USA 1991, 88, 9828-9832. [CrossRef]

52. Wu, D.H.; Wu, H.P.; Wang, C.S.; Tseng, H.Y.; Hwu, K.-K. Genome-wide InDel marker system for application in rice breeding and mapping studies. Euphytica 2012, 192, 131-143. [CrossRef]

53. Livak, K.J.; Schmittgen, T.D. Analysis of relative gene expression data using real-time quantitative PCR and the $2^{-\Delta \Delta \mathrm{Ct}}$ method. Methods 2001, 25, 402-408. [CrossRef] 
54. Dionisio-Sese, M.L.; Tobita, S. Antioxidant responses of rice seedlings to salinity stress. Plant Sci. 1998, 135, 1-9. [CrossRef]

55. Brennan, T.; Frenkel, C. Involvement of hydrogen peroxide in the regulation of senescence in pear. Plant Physiol. 1977, 59, 411-416. [CrossRef] [PubMed]

56. Giannopolitis, C.N.; Ries, S.K. Superoxide dismutases: I. Occurrence in higher plants. Plant Physiol. 1977, 59, 309-314. [CrossRef] [PubMed]

57. Maehly, A.C.; Chance, B. The assay of catalases and peroxidases. Methods Biochem. Anal. 1954, 1, $357-424$. [PubMed]

(C) 2019 by the authors. Licensee MDPI, Basel, Switzerland. This article is an open access article distributed under the terms and conditions of the Creative Commons Attribution (CC BY) license (http://creativecommons.org/licenses/by/4.0/). 